\section{LEADING THE WAY IN THE FIGHT AGAINST CAVITIES}

Breakthrough data published by Colgate has indicated that the use of a toothpaste containing 1.5\% arginine, an insoluble calcium compound, and 1,450 ppm fluoride, provides superior protection against caries, compared to toothpastes containing 1,450 ppm fluoride alone. Two two-year caries clinical studies, involving over 12,000 people, concluded that the use of a toothpaste containing 1.5\% arginine, an insoluble calcium compound and 1,450 ppm fluoride provided up to $20 \%$ fewer new cavities in two years versus the use of a regular (1,450ppm) fluoride toothpaste. ${ }^{1,2}$

These clinical findings support the launch of Colgate's latest innovation - Colgate Maximum Cavity Protection plus Sugar Acid Neutraliser toothpaste. The new everyday anti-cavity toothpaste contains Colgate's unique Sugar Acid Neutraliser technology, which is powered by $1.5 \%$ arginine and has been proven to help neutralise the damaging acids produced by the breakdown of dietary free sugars. ${ }^{3,4}$

Colgate Maximum Cavity Pro-

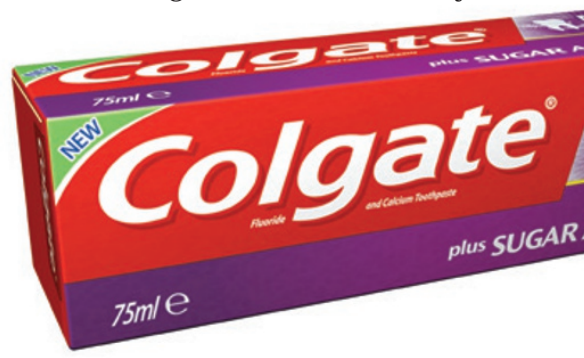
ern daily diets. ${ }^{5,6}$ younger children. 47: 582-590. Company, 2013. A): A45-54. 2013. 2009; 22: 3-8. tection plus Sugar Acid Neutraliser delivers a clinically proven and effective everyday anti-cavity solution, ideal for young families and children, helping to combat the ever increasing level of free sugar consumption found in mod-

For more information, including summaries of the clinical research conducted, visit: http://www. colgateprofessional.co.uk/products/ MaximumCavityProtection.

All variants in this new toothpaste range contain 1,450 ppm F, including a Kids variant which benefits from a milder mint flavour to make it more acceptable to

1. Kraivaphan P, Amornchat C, Triratana T et al. Two-year caries clinical study of the efficacy of novel dentifrices containing 1.5\% arginine, an insoluble calcium compound and 1,450 ppm fluoride. Caries Res 2013;

2. Hu D Y et al. Data on file. Colgate-Palmolive

3. Wolff $\mathrm{M}$, Corby $\mathrm{P}, \mathrm{Klaczany} \mathrm{G}$ et al. In vivo effects of a new dentifrice containing 1.5\% arginine and 1450 ppm fluoride on plaque metabolism. J Clin Dent 2013; 24(Spec Iss

4. Santarpia P, Lavender S, Gittens E et al. Submitted for publication in Am J Dent

5. Cummins D. Dental caries: a disease which remains a public health concern in the 21 st century - the exploration of a breakthrough technology for caries prevention. J Clin Dent 2013; 24[Spec Iss $A]: 1-14$

6. Bagramian R A, GarciaGodoy F, Volpe A R. The global increase in dental caries. A pending public health crisis. Am J Dent

\section{IMPLANT RESTORATION DRIVE}

Many dentists are recognising that dental implants should be the preferred option to replace missing teeth. 'R£LAX' training gives clinicians more confidence to discuss this treatment option with their patients. Attendees see for themselves how simple and profitable it is to restore the most common implant cases.

The 'R£LAX' initiative is helping dentists develop their practices. Completing the restora- tive phase of dental implant treatment makes good business sense. It improves clinical outcomes for patients and expands income options for dentists. The practice reputation is enhanced and the patient stays with their own dentist for long-term care.

DENTSPLY Implants is committed to improving access to implant treatment. To find an implant partner and attend an event, see www. dentists4implants.com/relax.

\section{MAKE TOOTHBRUSHING AN ENJOYABLE GAME}

Oral-B has recently presented their new Disney Magic Timer at a symposium at the $12^{\text {th }}$ Congress of the European Academy of Paediatric Dentistry in Poland. Using this app, kids are motivated in a playful way to complete two minutes of brushing. Each time they brush, Disney and Marvel character images are revealed during two minutes brushing time. Children have the opportunity to unlock 16 custom animations and 1,600 rewards, ensuring the app maintains its novelty and transforming the act of brushing into a game.

Paediatric specialists attending the event received this interactive oral care app for children with great enthusiasm, as it has already been shown to increase brushing time, which is identified by experts as an area of concern amongst children. It is available for free in many countries and languages worldwide (Disney Magic Timer by Oral-B, available on iOS and Google Play). It is hoped that such new technology will help to positively influence children's oral health and will, as a consequence, reduce the incidence of carious lesions and lower DMF levels.

\section{THE IDEAL TOOL FOR PERIODONTAL EXAMS}

The Florida Probe from Clark Dental will transform the way you conduct periodontal exams.

Providing increased productivity, improved accuracy, automatic charting, and easy customisation, the Florida Probe lets even complex examinations be completed in as little as ten minutes.

With only one examiner needed to carry out an exam thanks to the revolutionary new VoiceWorks voice controlled charting system, the Florida Probe allows far greater accuracy and reliability. You will instantly see the benefits as the new system generates a more efficient workflow within your practice.

The greater precision of $0.2 \mathrm{~mm}$ will also improve the exactness of your measurements, which will aid in determining the correct diagnosis and follow-up for each patient. No longer will you need to try and read the thin bands on a regular periodontal probe. Simply tap the footswitch and your numerical data is recorded.

The Florida Probe is the ideal tool for conducting a periodontal exam and will prove advantageous to any practice. To find out more, contact the team at Clark Dental today or visit the website for more information.

Call Clark Dental on 01268733 146, emailinfo@clarkdental. co.uk or visit www.clarkdental.co.uk.

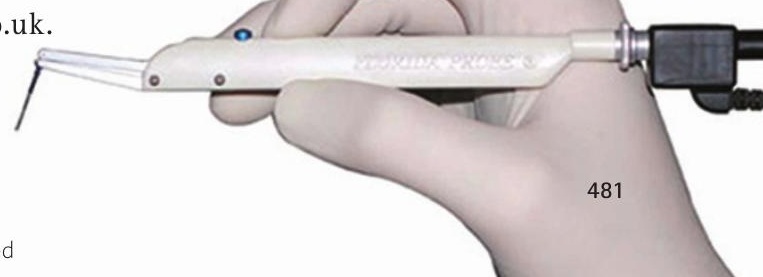

